\title{
MIXED REALITY AS ONE OF MAIN COMPONENTS OF THE SCIENTIFIC GATEWAY
}

\author{
Eva Pajorová and Ladislav Hluchý \\ Institute of Informatics, Slovak Academy of Sciences \\ Bratislava, Slovakia
}

\begin{abstract}
Data visualization and mixed reality is incredibly important as a tool of communication - whether for hard or softer data. A Science Gateway is a connection between scientists and their computational tools in the form of web portal. It creates a space for communities, collaboration and data sharing and visualization in a comprehensive and e-client manner. The main purpose of such a solution is to allow users to access the computational resources, process and analyze their data and get the results in a uniform and user friendly way. It is very important for us, researcher to communicate our science to non-scientists. Our paper deal with position the mixed reality as a one of main component the scientific gateway and portals. The scientific portals and gateways are cumulative all classes of visualization and Mixed reality.
\end{abstract}

\section{KEYWORDS}

Mixed Reality, Scientifics Gateway, Research Results, 3D Visualization

\section{INTRODUCTION}

Science gateways provide access to advanced resources for science and engineering researchers, educators, and students. Through streamlined, online, user-friendly interfaces, gateways combine a variety of cyberinfrastructure $(\mathrm{CI})$ components in support of a community-specific set of tools, applications, and data collections. In general, these specialized, shared resources are integrated as a Web portal, mobile app, or a suite of applications. Through science gateways, broad communities of researchers can access diverse resources which can save both time and money for themselves and their institutions. Mixed reality is used as an independent concept, or to classify the spectrum of reality technologies. The area between the two extremes, where both the real and the virtual are mixed is called mixed reality. This in turn is said to consist of both augmented reality, where the virtual augments the real, and augmented virtuality, where the real augments the virtual. Augmented reality (AR) supplements the real world with virtual objects, such that virtual objects appear to coexist in the same space as the real world. Mixed reality called as mixed because it is regarded as a system in which augmented reality (AR) and virtual reality (VR) technologies are combined. For example some experts present the framework for mixed reality agents, i.e. agents that exist in both the real and virtual space. These agents combine the physical presence of a robot with the adaptability and expressivity of a virtual character. The architecture can be employed in the context of a mobile collaborative mixed reality environment that is cohabited by both robots and humans. As an example application they applied the framework to a museum virtual presence of the mixed reality agent to convey an individual and personalized learning experience. Through user-friendly web interfaces integrated into the same environment, researchers and scientists can securely and transparently access to computational and data sources, services, tools, sensors, etc. Scientific portals and gateways are able to provide a community-centric view, workflow/dataflow services and a strong support in accessing to the cyber infrastructure including grid and cloud based resources. In each of Science contexts, scientific gateways play a key role since they allow scientists to transparently access to distributed data repositories (across several domains and institutions), metadata sources, carry out search \& discovery activities, as well as visualization and mixed reality and analysis ones, etc. Finally, Scientific Gateways can play an important role for training students (at the academic level) in the different sciences disciplines, attract new users and representing a relevant centralized knowledge repository in the Sciences context. 
Currently they are lot of communications framework and gateways. For example Story telling (Kosara, 2017) is an art and free form mostly used as entertainment in the arts. The framework of storytelling can be applied very effectively to scientific data to make it intelligible to those learning and those who are not scientists. To allow users to interact remotely with future supercomputers and large-scale computing environments in a more interactive and visual manner we present a tool called Vine Toolkit that has been successfully used as a core web platform for various gateways (Song, 2016). Also Vine Toolkit is a modular, extensible and easy-to-use tool as well as high-level Application Programming Interface (API) for various applications, visualization components and building blocks to allow interoperability between many different HPC and grid technologies.

P-GRADE is a good example of highly integrated parallel application development web-based system for Grid and clusters (P-GRADE Grid Portal). It uses Globus, Condor-G, ARC, BOINC and MPICH-G2 as grid-aware middleware to conduct computations. The framework supplies a repository of gems enabling scripts to interface low-level resources such as PBS queues, EGEE computing elements, LFC directories and other types of Grid resources. Moreover, GridSpace2 provides a Web 2.0-based Experiment Workbench supporting joint development and execution of virtual experiments by groups of collaborating scientists. GridSpace 2 main idea to enable HPC environments via web interface is very similar to Vine Toolkit main idea, however GridSpace with its scripts is more like programming environment for the Grid \{suitable for advanced users or programmers; on the other hand, Vine Toolkit Applications do not require from the user knowledge of scripting languages and focus on delivering ready to use GUIs. Moreover, thanks to Vine Toolkit native JSDL and BES support and the QosCosGrid middleware stack used in the prototype portal can be easily changed to any type supported by Vine middleware's without changes in code or even the need to restart the portal. Our contribution is in Astro Portal: A Science Gateway for Large-scale Astronomy Data Analysis. We have developed mixed reality visualization tools. One of them is mixed reality visualization tool of dynamical evolution of Oort cloud as a part of Protoplanetary disk. Second our contribution is in Water HUB is a Science Gateway for Water Research and Education when we are working on mixed reality tools for water management?

\section{MIXED REALITY (MR) AS A WAY OF REPRESENTATIONS OF DATASETS}

There are some main reasons for that scientist are using VR as visual representations of datasets.

1. For visual control in High performance computing (HPC) of the execution proces

2. For presentations the research academic results in different disciplines

3. For publication of research results

4. For scientists and non-scientist, students as a directly visual education form

\subsection{MR as Visual Control of the Execution Process}

MR and visualization have the main position as a way to control execution process. The visualization tool is designed as a plug in module. Client asking for visualization is as a "visualization client". Output data on the storage element are the inputs data for visualization jobs. Visualization workers are to modify data to the visualizable formats, but also to prepare the typical visualization scenes. 


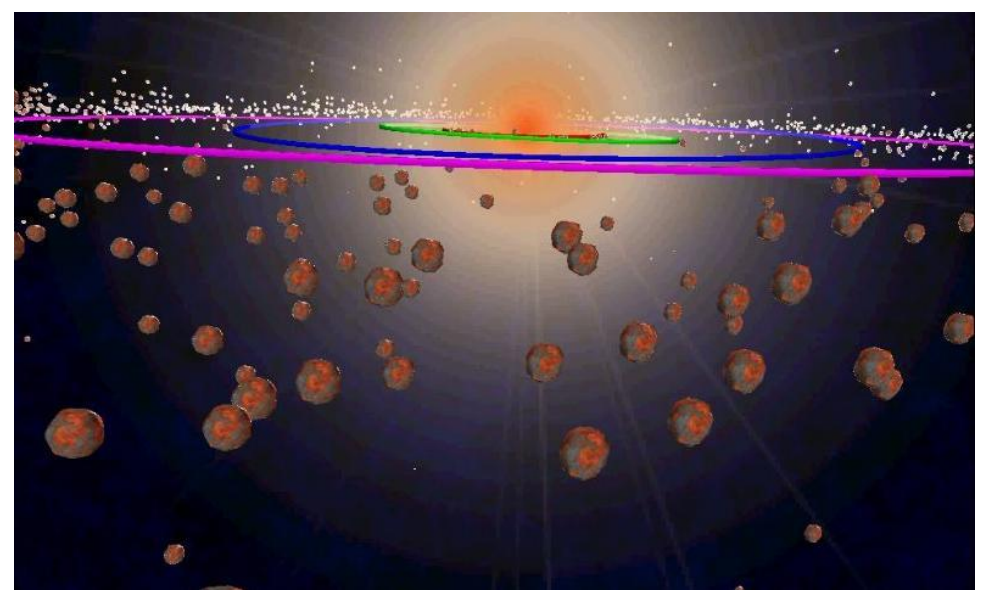

Figure 1. Example when is MR and Visualization using as a Control of Execution Process

\subsection{MR Tools as a Presentations Form}

The Science Gateways Research Center develop and use open source software to create user tools and environments that help scientific communities do what they could not otherwise do. The Science Gateways Research Center researches, develops, and operates science gateways in collaboration with many clients and partners. Advance in sciences and engineering has put high demand on tools for high-performance large-scale visual data exploration and analysis.

\subsection{Formal Publication of Research Results}

The goal of MR and scientific visualization is to help scientists view and better understand their large data. (Šipková, 2016).There are data from computing, experiments or numerical simulations. Directly the output data are difficult to understand. MR and scientific visualization can help with these difficulties. Using mixed reality techniques, the data can be viewed naturally. Viewing the data as MR can quickly draw the scientist's attention to interesting and/or anomalous portions of the data. Scientists to use MR from the beginning to end of their experiments and simulations. This also allows the scientists to develop a set of MR tools and techniques that will help them understand their data. We have developed lot of MR tool in different disciplines for better understand the research results. Such as MR tool for natural disasters, VR tool for astronomical research, MR tool for water management and so on.

For example VR tool for water management are using for better understand the process of water simulation in different research goals (Pajorová, 2016). Our MR tool is composed from 5 modules which all have different mission. They can work separately or together as a one framework. See some outputs from MR tool for water management. Water HUB is a Science Gateway for Water Research and Education, is a global communication and research platform for research, education, knowledge sharing and global outreach (Cuckerberk, 2017). Built on the HUB zero cyberinfrastructure for scientific collaboration, Water HUB develops and makes available modeling and data sharing tools used in water related research and education activities. Among its tools, the SWAT Share online application not only allows users to run and calibrate their SWAT models, it also supports sharing of models and output data visualization through web browsers. 


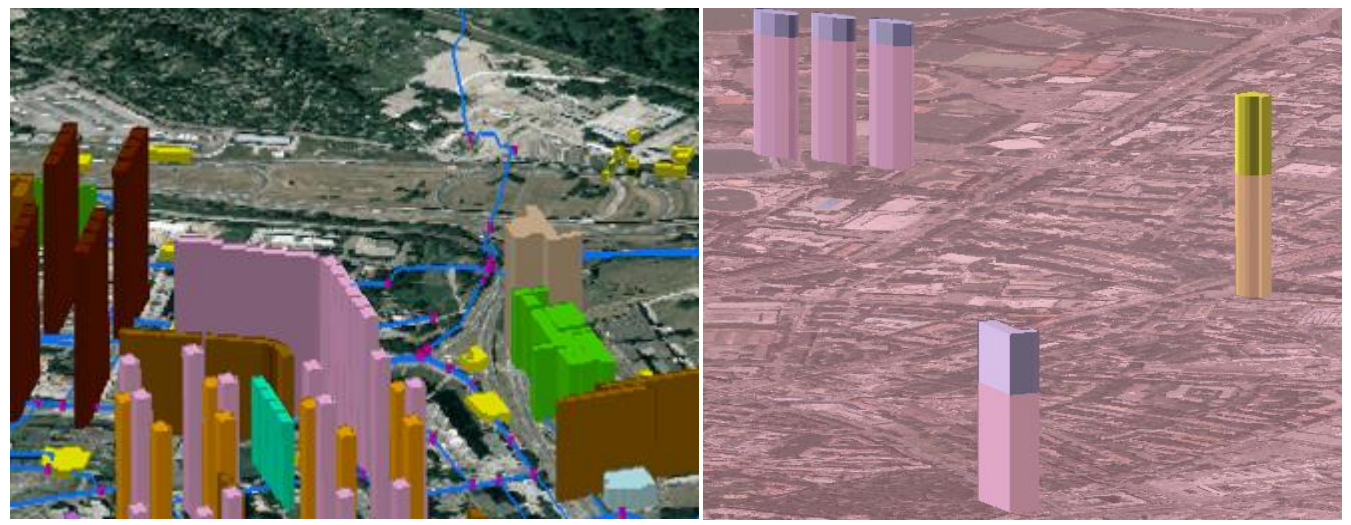

Figure 2. Pictures Shows the Water Simulation Research Results

\subsection{MR Tools as a Visual Teaching and Education Form}

Educational visualization is using a simulation normally created on a computer to create an image of something so it can be taught about. This is very useful when teaching about a topic which is difficult to otherwise see, for example, atomic structure, because atoms are far too small to be studied easily without expensive and difficult to use scientific equipment. It can also be used to view past events, such as looking at dinosaurs, or looking at things that are difficult or fragile to look at in reality like the human skeleton, without causing physical or mental harm to a subjective volunteer or cadaver.

\section{CONCLUSION}

The goal of our paper is to demonstrate the some examples and to support the mixed reality and visualization as an essential component in new portals and gateways technologies. For a future we want to create a scientific gateway for some scientific disciplines as are naturel disasters sciences with all visualization aspects by using mixed reality and all new one visualization technologies.

\section{ACKNOWLEDGEMENT}

This work is supported by projects VEGA 2/0167/16 and U - COMP: APVV - 17-0619.

\section{REFERENCES}

Cuckerberk, M. F., 2017. How the WaterHub Works http://www.campserv.emory.edu/fm/energy_utilities/waterhub/how\%20it\%20works. https://www.facebook.com/zuck/posts/10101319050523971.

Kosara, R. and Mackinlay, J., 2017. Storytelling: The Next Step for Visualization. Computer (Special Issue on CuttingEdge Research in Visualization https://www.bing.com/search?q=Story+telling+papers\&form=PRUSEN\&pc=UE07\&mkt=enus\&httpsmsn=1\&refig=0fdab404c62d411d80bb6139a1549183\&sp=-1\&pq=story+telling+papers\&sc=7$20 \& q s=n \& s k=\& c v i d=0 f d a b 404 c 62 d 411 \mathrm{~d} 80 \mathrm{bb} 6139 \mathrm{a} 1549183)$.

Pajorová,, E. and Hluchy, L., 2016. Water management - 3D visualization of research results. In Inovatívne informačno-komunikačné technológie vo vodnom hospodárstve. - Bratislava: STU Bratislava, p. 53-58. ISBN 978-80-89535-19-4.

Song, C., Zhao, L. and Biehl, L., 2016. New Science Gateways for Advanced Computing Simulations and Visualization Using Vine Toolkit in Pl-Grid.

Šipková, V., Hluchý, L., Dobrucký, M., Bartok, J., Nguyen, B. M., 2016. Manufacturing of weather forecasting simulations on high performance infrastructures. In ECW 2016 Environmental Computing Workshop: 12th International IEEE Conference on eScience. - Baltimore, USA: IEEE, 2016, p. 432-439. ISBN 978-1-5090-4273-9. ISSN 2325-372X. 